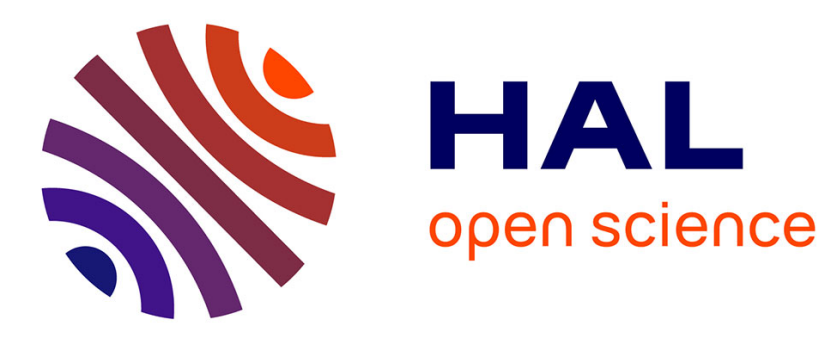

\title{
Annexes statistiques
}

- [.]revue Forestière Française, Rédaction

\section{- To cite this version:}

- [.]revue Forestière Française, Rédaction. Annexes statistiques. 1985, pp.228-247. 10.4267/2042/21806 . hal-03535077

\section{HAL Id: hal-03535077 https://hal.science/hal-03535077}

Submitted on 19 Jan 2022

HAL is a multi-disciplinary open access archive for the deposit and dissemination of scientific research documents, whether they are published or not. The documents may come from teaching and research institutions in France or abroad, or from public or private research centers.
L'archive ouverte pluridisciplinaire HAL, est destinée au dépôt et à la diffusion de documents scientifiques de niveau recherche, publiés ou non, émanant des établissements d'enseignement et de recherche français ou étrangers, des laboratoires publics ou privés. 


\section{ANNEXES STATISTIQUES}

Annexe 1 Superficie, volume sur pied, accroissement par essence.

Annexe II Surface des forêts par catégories de propriétaires.

Annexe III Production des branches: Exploitation forestière et scierie en 1983.

Annexe IV Evolution de la production de sciages de 1947 à 1983.

Annexe $\mathbf{V} \quad$ Valeurs hors taxes de la récolte française.

Annexe VI Place de la France dans la C.E.E. et dans le monde pour la production de bois et dérivés en 1982.

Annexe VII Réception de bois dans les industries (pâtes et papiers - panneaux de particules contreplaqués).

Annexe VIII Activité d'exploitation forestière.

Répartition des entreprises selon la récolte en 1983.

Annexe IX Activité de scierie.

Répartition des entreprises selon la production en 1983.

Annexe $X$ Activités de seconde transformation.

Secteur travail mécanique du bois.

Annexe XI Activités de seconde transformation.

Secteur ameublement.

Annexe XII Cartes de localisations des scieries feuillues

des scieries résineuses

Annexe XIII Activités du secteur pâtes et papiers.

Annexe XIV Carte de localisation des usines de panneaux, de pâtes et papiers cartons.

Annexe XV Commerce extérieur du bois en 1984.

Annexe XVI Bilan matière-bois en 1982.

Annexe XVII Liste d'adresses. 


\begin{tabular}{|c|c|c|c|}
\hline $\begin{array}{c}\text { Essences } \\
\text { prédominantes }\end{array}$ & $\begin{array}{l}\text { Superficie forêt } \\
\text { (1 } 000 \text { ha) }\end{array}$ & $\begin{array}{l}\text { Volume sur pied } \\
\text { (1000 } \mathrm{m}^{3} \text { sur écorce) }\end{array}$ & $\begin{array}{c}\text { Accroissement } \\
\text { (1000 } \mathrm{m}^{3} \text { sur écorce) }\end{array}$ \\
\hline $\begin{array}{l}\text { Chêne pédonculé } \\
\text { et rouvre } \ldots \ldots .\end{array}$ & 4143,0 & 402440 & 9551,6 \\
\hline Hêtre ............. & 1237,5 & 202312 & 4775,4 \\
\hline Châtaignier ........ & 558,5 & 73321 & 2996 \\
\hline Autres feuillus ....... & 2666,0 & 295282 & 10991 \\
\hline TOTAL FEUILLUS ..... & 8606,0 & 973355 & 28314 \\
\hline Sapin et Epicèa .... . & 1200,5 & 259758 & 7453,8 \\
\hline Pin maritime $\ldots . .$. & 1345,0 & 157258 & 9608,8 \\
\hline Autres Pins $\ldots \ldots \ldots$ & 1613,0 & 173330 & 6240,5 \\
\hline Autres conifères ..... & 363,0 & 29577 & 1541,6 \\
\hline TOTAL CONIFĖRES ... & 4521,5 & 619923 & 24844,7 \\
\hline $\begin{array}{l}\text { TOTAL GÉNÉRAL } \\
\text { Feuillus + conifères }\end{array}$ & 13127,5 & 1593278 & 53158,7 \\
\hline $\begin{array}{l}\text { Peupleraies ......... } \\
\text { Equivalent arbres } \\
\text { et bosquets épars } \ldots .\end{array}$ & $\begin{array}{r}265,0 \\
1040,0\end{array}$ & & \\
\hline
\end{tabular}

\section{ANNEXE}

Surface des forêts par catégories de propriétaires

(en milliers d'hectares)

\begin{tabular}{|c|c|}
\hline 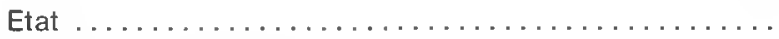 & 1437 \\
\hline 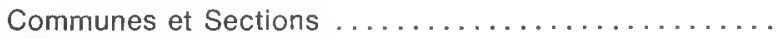 & 2582 \\
\hline 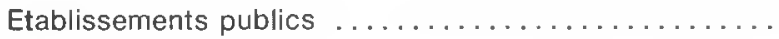 & 160 \\
\hline 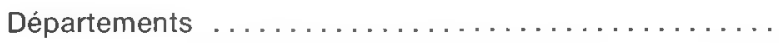 & 21 \\
\hline Propriètaires privés $\ldots \ldots \ldots \ldots \ldots \ldots$ & 10920 \\
\hline 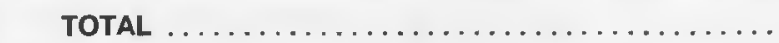 & 15120 \\
\hline
\end{tabular}




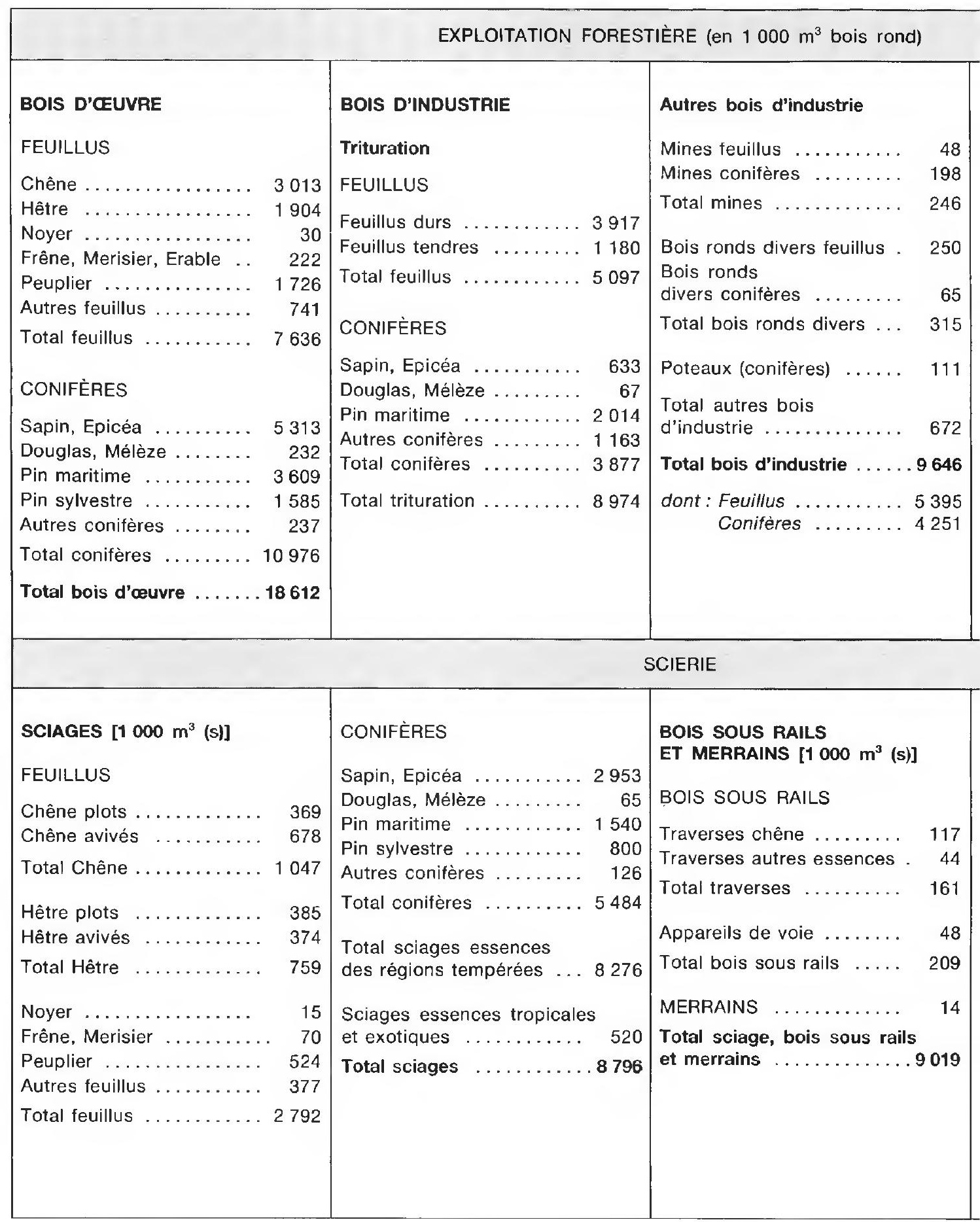




\section{BOIS DE FEU}

Bois de feu

non auto-consommé .... 1232

Bois ronds

pour carbonisation ...... 147

Total bois de feu

commercialisé ......... 1379

TOTAL GÉNÉRAL

29637

\section{CHUTES DE SCIERIE}

(1000 tonnes)

\section{TRITURATION PLAQUETTES}

Feuillus ............. 302

Conifères ........... 691

Total . . . . . . . . . . . . . . 993

Chutes brutes ........ 1389

Total trituration ......... 2382

Chutes pour

autres utilisations ....... 414

Total chutes de scierie . . 2796

\section{Sciures}

commercialisées ........ 533

Total produits connexes

de scierie .......... 3329
29,6 millions de $\mathrm{m}^{3}$ produits par nos exploitations forestières en 1983

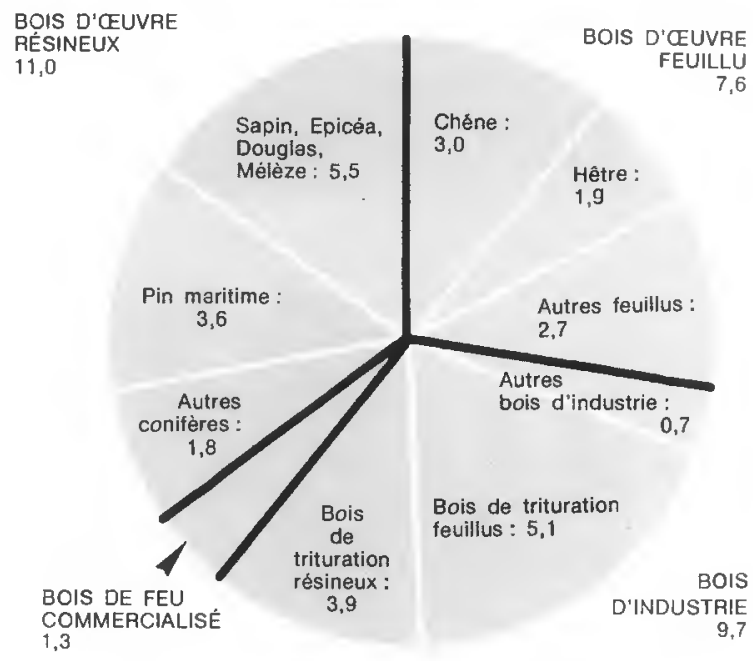

A cela, if faut ajouter le bois auto-consommé n'empruntant pas les circuits habituels de la commercialisation. Son volume pourrait atteindre 10 millions de $\mathrm{m}^{3}$.

en millions de $\mathrm{m}^{3}$

9 millions de $\mathrm{m}^{3}$ produits

par nos scieries en 1983

SCIAGES

RÉSINEUX : 5,5

SCIAGES

FEUILLUS: $\quad$ Sapin, Epicéa,

3,2 Douglas, Mélèze: 3,0

CHUTES DE SCIERIES

COMMERCIALISEES :

Autres

2,8 millions
de tonnes

Che̊ne: 1,0

résineux: 0,9

Hêtre: 0,8

Peuplier : 0,5

Autres feuillus

tempérés : 0,4

Feuillus tropicaux : 0.5

BOIS SOUS RAILS

ET MERRAINS : 


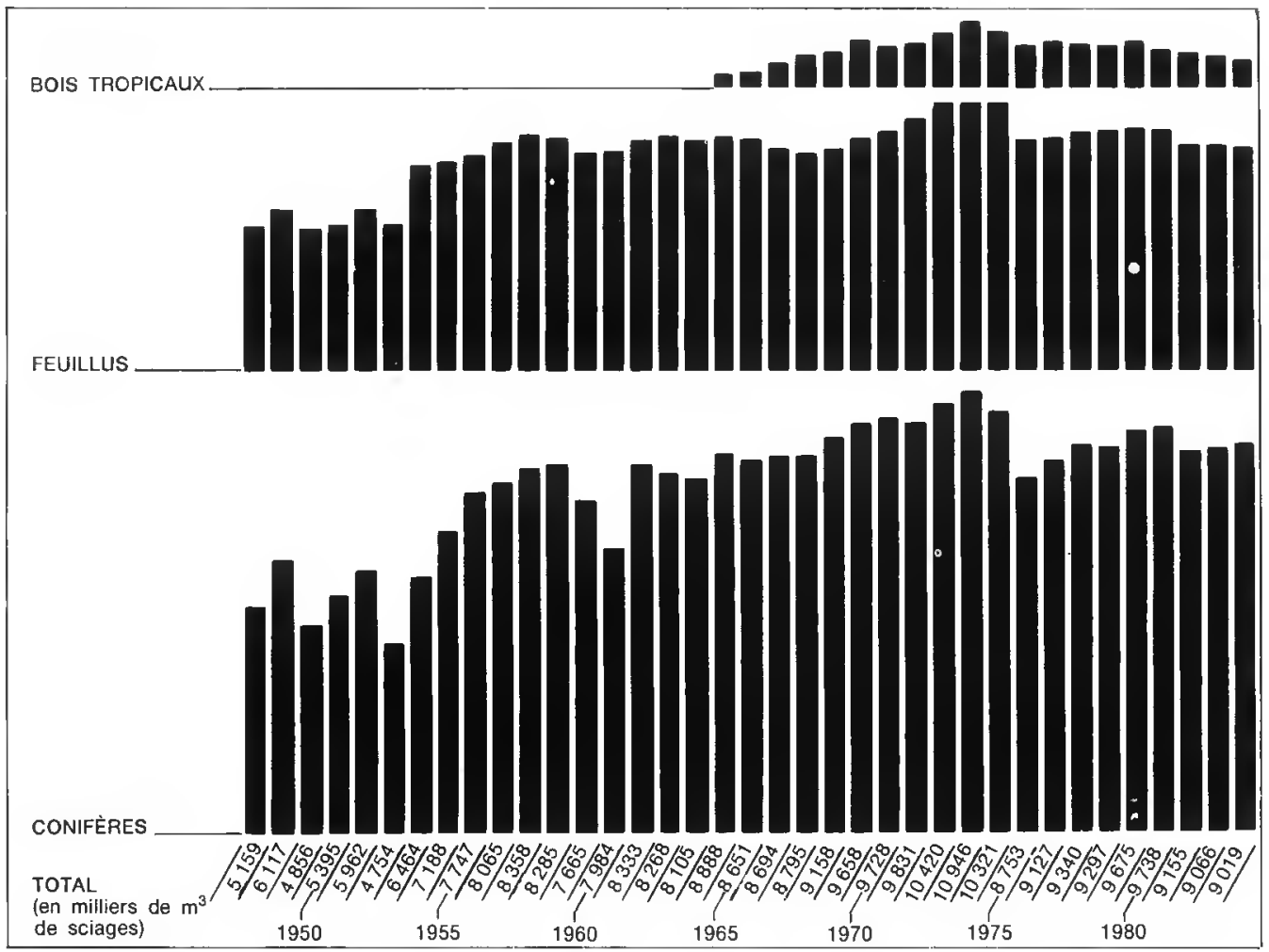

ANNEXE $V$

Valeurs hors taxes de la récolte française (en milliers de francs)

\begin{tabular}{|c|c|c|c|c|c|}
\hline \multicolumn{5}{|c|}{ Valeurs H.T. des bois sur pied livrés en 1982 à l'exploitation forestière } \\
\hline Bois d'œuvre & $\begin{array}{c}\text { Bois } \\
\text { d'industrie }\end{array}$ & Bois de feu & $\begin{array}{c}\text { Bois ronds } \\
\text { pour } \\
\text { carbonisation }\end{array}$ & Total & $\begin{array}{c}\text { dont forêts } \\
\text { soumises }\end{array}$ \\
\hline 4741233 & 368038 & 48272 & 2125 & 5159668 & 1813808 \\
\hline
\end{tabular}

\begin{tabular}{|c|c|c|c|c|c|}
\hline \multicolumn{7}{|c|}{ Valeurs H.T. des bois enlevés en 1983 } & \multirow{2}{*}{$\begin{array}{c}\text { Valeurs } \\
\text { des sciages } \\
\text { Bois d'œuvre }\end{array}$} & $\begin{array}{c}\text { Bois } \\
\text { d'industrie }\end{array}$ & Bois de feu & $\begin{array}{c}\text { Bois ronds } \\
\text { pour } \\
\text { carbonisation }\end{array}$ & Total & $\begin{array}{c}\text { colisés } \\
\text { en }\end{array} 6443169$ & 1242291 & 207068 & 15083 & 7907611 & 9953404 \\
\hline
\end{tabular}


Place de la France dans la C.E.E. et dans le Monde

Production de bois et dérivés en 1982

\begin{tabular}{|c|c|c|c|c|c|c|}
\hline & $\begin{array}{c}\text { Bois } \\
\text { d'ceuvre } \\
\left(1000 \mathrm{~m}^{3}\right)\end{array}$ & $\begin{array}{l}\text { Bois } \\
\text { de trituration } \\
\left(1000 \mathrm{~m}^{3}\right)\end{array}$ & $\begin{array}{l}\text { Sciages } \\
\text { et traverses } \\
\left(1000 \mathrm{~m}^{3}\right)\end{array}$ & $\mid \begin{array}{c}\text { Panneaux } \\
\text { à base de bois } \\
\left(1000 \mathrm{~m}^{3}\right)\end{array}$ & $\begin{array}{c}\text { Pâtes } \\
\text { de bois } \\
(1000 \text { t) }\end{array}$ & $\begin{array}{l}\text { Papiers } \\
\text { et cartons } \\
(1000 \text { t) }\end{array}$ \\
\hline Danemark ... & 2016 & 287 & 829 & 280 & 57 & 229 \\
\hline R.F.A. $\ldots \ldots$ & 12050 & 9630 & 8715 & 6295 & 2021 & 7796 \\
\hline Grèce ...... & 608 & 132 & 363 & 460 & 21 & 266 \\
\hline France ..... & 17912 & 8509 & 9058 & 2701 & 1843 & 5067 \\
\hline Irlande ..... & 547 & 480 & 238 & 42 & 一 & 20 \\
\hline Italie ....... & 1449 & 917 & 2351 & 3154 & 704 & 4323 \\
\hline $\begin{array}{l}\text { Belgique } \\
\text { Luxembourg }\end{array}$ & 1510 & 570 & 671 & 1755 & 358 & 864 \\
\hline Pays-Bas .... & 180 & 332 & 204 & 104 & 200 & 1590 \\
\hline Royaume-Uni & 2950 & 1168 & 1687 & 653 & 144 & 3186 \\
\hline Total C.E.E. & 39222 & 22025 & 24116 & 15444 & 5348 & 23341 \\
\hline
\end{tabular}

\begin{tabular}{|c|c|c|c|c|c|c|}
\hline Espagne .... & 4769 & 6638 & 2854 & 1675 & 1282 & 2684 \\
\hline Portugal .... & 3912 & 3280 & 2229 & 491 & 685 & 485 \\
\hline Norvège .... & 4610 & 4030 & 2262 & 608 & 1523 & 1305 \\
\hline Suède .... & 22385 & 23725 & 11212 & 1503 & 7706 & 5928 \\
\hline Finiande .... & 15130 & 18680 & 7356 & 1489 & 6714 & 5895 \\
\hline U.R.S.S. ... & 152000 & 37700 & 96000 & 10980 & 9067 & 9098 \\
\hline U.S.A. .... & 161220 & 122285 & 61110 & 23000 & 44833 & 55064 \\
\hline Canada .... & 93675 & 41335 & 37173 & 3284 & 17318 & 12407 \\
\hline Japon .... & 20396 & 10383 & 32537 & 8836 & 8563 & 17368 \\
\hline
\end{tabular}

Source: F.A.O. 


\begin{tabular}{|c|c|c|c|c|c|c|}
\hline $\begin{array}{l}\text { en milliers de stères } \\
\text { coefficients de conversion } \\
\text { Pin maritime : } 1 \mathrm{t}=2,3 \text { stères } \\
\text { Châtaignier : } 1 \mathrm{t}=2 \text { stères }\end{array}$ & 1978 & 1979 & 1980 & 1981 & 1982 & 1983 \\
\hline $\begin{array}{l}\text { RÉCEPTION DE BOIS } \\
\text { Bois trançais }\end{array}$ & & & & & & \\
\hline $\begin{array}{l}\text { Sapin - Epicéa } \ldots \ldots \ldots \ldots \ldots \ldots \\
\text { Pin sylvestre } \ldots \ldots \ldots \ldots \ldots \\
\text { Pin maritime } \ldots \ldots \ldots \ldots \ldots \ldots \\
\text { Total résineux } \ldots \ldots \ldots \ldots \ldots \ldots \\
\text { dont délignures } \ldots \ldots \ldots \ldots \ldots\end{array}$ & \begin{tabular}{ll|}
1 & 193,5 \\
1 & 414,4 \\
2 & 447,9 \\
5 & 055,8 \\
2 & 163,5
\end{tabular} & $\begin{array}{ll}1 & 160,2 \\
1 & 508,4 \\
2 & 302 \\
4 & 970,6 \\
2 & 214,9\end{array}$ & $\begin{array}{ll}1 & 088,4 \\
1 & 739 \\
2 & 487,8 \\
5 & 315,2 \\
2 & 255,1\end{array}$ & $\begin{array}{ll}1 & 090,1 \\
1 & 678,2 \\
2 & 809,7 \\
5 & 578 \\
2 & 250,4\end{array}$ & $\begin{array}{ll}1 & 225,2 \\
1 & 589,7 \\
2 & 629,7 \\
5 & 444,6 \\
2 & 150,9\end{array}$ & $\begin{array}{l}1276,0 \\
2065,5 \\
2927,4 \\
6268,9 \\
2405,8\end{array}$ \\
\hline 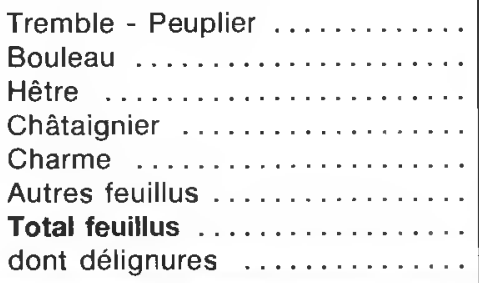 & $\begin{array}{r}391,5 \\
227,3 \\
728,7 \\
374,7 \\
199,2 \\
2594,5 \\
4515,9 \\
497,2\end{array}$ & $\begin{array}{r}376,5 \\
207,2 \\
582,8 \\
368 \\
177,3 \\
2639,3 \\
4351,1 \\
440,8\end{array}$ & $\begin{array}{r}436,2 \\
270,6 \\
543,3 \\
384,6 \\
184,6 \\
2421,4 \\
4240,7 \\
477,1\end{array}$ & \begin{tabular}{r|}
271,3 \\
294,8 \\
423 \\
408,8 \\
173,3 \\
2244,2 \\
3815,4 \\
489,3
\end{tabular} & $\begin{array}{r}270,6 \\
325,6 \\
422,2 \\
383,7 \\
175,3 \\
2189,2 \\
3766,6 \\
490,8\end{array}$ & $\begin{array}{r}265,5 \\
249,8 \\
424,4 \\
354,2 \\
174,7 \\
2251,0 \\
3719,6 \\
446,2\end{array}$ \\
\hline $\begin{array}{l}\text { inçais } \ldots \ldots \ldots \ldots \ldots \\
\text { es } \ldots \ldots \ldots \ldots \ldots\end{array}$ & $\begin{array}{l}9571,7 \\
2660,7\end{array}$ & $\begin{array}{l}9321,7 \\
2655,7\end{array}$ & $\begin{array}{l}9555,9 \\
2732,2\end{array}$ & $\begin{array}{l}9393,4 \\
2739,7\end{array}$ & $\begin{array}{l}9211,2 \\
2641,7\end{array}$ & $\begin{array}{l}9988,5 \\
2852,0\end{array}$ \\
\hline $\begin{array}{l}\text { Bois importés } \\
\text { Sapin - Epicéa } \ldots \ldots \ldots \ldots \ldots \ldots \\
\text { Pin sylvestre } \ldots \ldots \ldots \ldots \ldots \ldots \\
\text { Pin maritime } \ldots \ldots \ldots \ldots \ldots \ldots \\
\text { Tremble } \ldots \ldots \ldots \ldots \ldots \ldots \\
\text { Hêtre } \ldots \ldots \ldots \ldots \ldots \ldots \\
\text { Charme et autres feuillus } \ldots \ldots\end{array}$ & $\begin{array}{c}805 \\
2,1 \\
- \\
- \\
6,7 \\
0,4\end{array}$ & $\begin{array}{l}766,3 \\
308,5 \\
- \\
10,5 \\
21\end{array}$ & $\begin{array}{l}791,2 \\
416 \\
- \\
6,4 \\
13,6 \\
128,9\end{array}$ & $\begin{array}{r}670,3 \\
158,1 \\
- \\
4,4 \\
5,7 \\
39,9\end{array}$ & $\begin{array}{r}573,8 \\
50,2 \\
- \\
9,8 \\
16,3 \\
22,8\end{array}$ & $\begin{array}{r}330,5 \\
11,7 \\
- \\
31,5 \\
10,0 \\
40,8\end{array}$ \\
\hline $\begin{array}{l}\text { Total bois in } \\
\text { dont délignt }\end{array}$ & $\begin{array}{l}814,2 \\
206\end{array}$ & $\begin{array}{r}1106,3 \\
220,9\end{array}$ & $\begin{array}{r}1356,1 \\
524,2\end{array}$ & $\begin{array}{l}878,4 \\
327,1\end{array}$ & $\begin{array}{l}672,9 \\
269,0\end{array}$ & $\begin{array}{l}424,5 \\
229,1\end{array}$ \\
\hline $\begin{array}{l}\text { Total des réceptions de bois } \\
\text { Résineux } \ldots \ldots \ldots \ldots \ldots \ldots \ldots \\
\text { dont délignures } \ldots \ldots \ldots \ldots \ldots\end{array}$ & $\begin{array}{l}5862,9 \\
2362,5\end{array}$ & $\begin{array}{l}6045,4 \\
2429,4\end{array}$ & $\begin{array}{l}6522,4 \\
2771,7\end{array}$ & $\begin{array}{l}6406,4 \\
2571,3\end{array}$ & $\begin{array}{l}6068,6 \\
2401,3\end{array}$ & $\begin{array}{l}6611,1 \\
2603,2\end{array}$ \\
\hline $\begin{array}{l}\text { Feuillus } \ldots \ldots \ldots \ldots \ldots \ldots \ldots \\
\text { dont délignures } \ldots \ldots \ldots \ldots \ldots\end{array}$ & $\begin{array}{l}4523 \\
504,2\end{array}$ & $\begin{array}{r}4382,6 \\
447,2\end{array}$ & $\begin{array}{r}4389,6 \\
484,6\end{array}$ & $\begin{array}{r}3865,4 \\
495,5\end{array}$ & $\begin{array}{r}3815,5 \\
509,4\end{array}$ & $\begin{array}{r}3801,9 \\
477,9\end{array}$ \\
\hline $\begin{array}{l}\text { Total des réceptions } \\
\text { dont délignures ..... }\end{array}$ & $\begin{array}{r}10385,9 \\
2866,7\end{array}$ & $\begin{array}{l}10428 \\
2876,6\end{array}$ & $\begin{array}{l}10912 \\
3256,4\end{array}$ & $\begin{array}{r}10271,8 \\
3066,8\end{array}$ & $\begin{array}{l}9884,1 \\
2910,7\end{array}$ & $\begin{array}{r}10413,0 \\
3081,1\end{array}$ \\
\hline
\end{tabular}

\section{ORIGINE DES BOIS IMPORTÉS}

Canada

U.R.S.S.

U.S.A. ..................

Autres pays

\begin{tabular}{l|l} 
& \\
& \\
206 & 219 \\
431 & 359 \\
139 & 149 \\
- & 151 \\
38 & 228
\end{tabular}
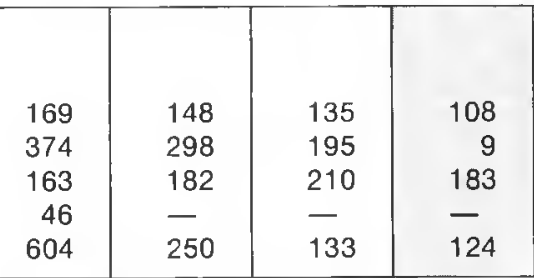

Source: Fédèration française des pâtes à papier 
En milliers de tonnes

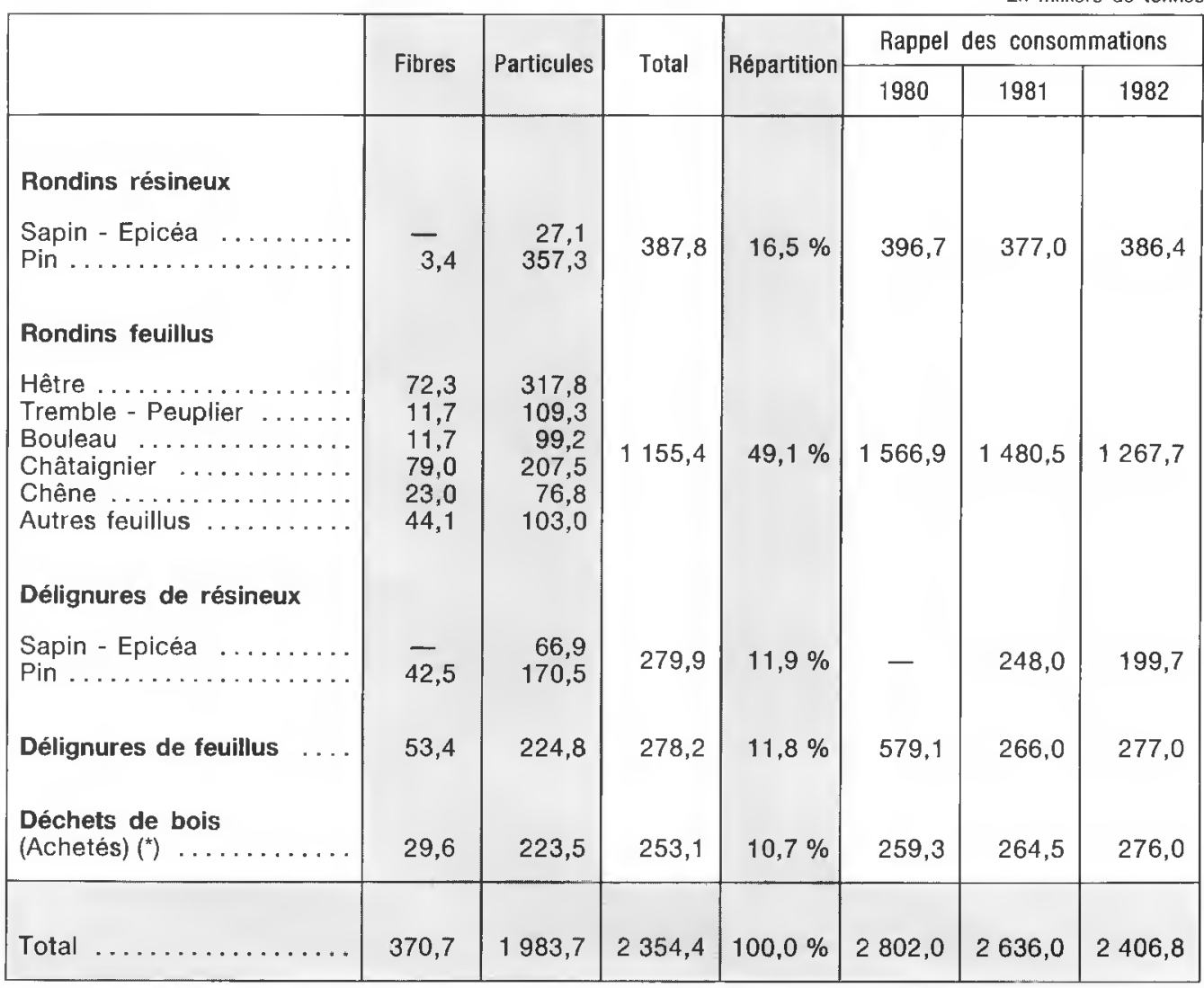

(") Y compris plaquettes.

ANNEXE VIl c Réception de bois dans l'industrie des contreplaqués

\begin{tabular}{|c|c|c|c|c|c|}
\hline \multirow[t]{2}{*}{ Années } & \multirow[t]{2}{*}{ Produit } & \multirow{2}{*}{$\begin{array}{l}\text { Production } \\
\left(\mathrm{m}^{3}\right)\end{array}$} & \multicolumn{3}{|c|}{$\begin{array}{l}\text { Estimation des consommations de bois } \\
\left.\text { (bois rond) (en } \mathrm{m}^{3} \mathrm{EQ}\right)\end{array}$} \\
\hline & & & Total & Résineux & Feuillus \\
\hline 1982 & $\begin{array}{l}\text { Contreplaqués, total ... } \\
- \text { multiplis } \ldots \ldots \ldots \\
\text { - lattes ............. }\end{array}$ & $\begin{array}{r}477000 \\
428000 \\
49000\end{array}$ & $\begin{array}{c}900000 \\
- \\
-\end{array}$ & $\begin{array}{c}210000 \\
- \\
-\end{array}$ & $\begin{array}{c}690000 \\
- \\
-\end{array}$ \\
\hline 1983 & $\begin{array}{l}\text { Contreplaqués, total ... } \\
- \text { multiplis } \ldots \ldots \ldots \ldots \\
\text { - lattes ............ }\end{array}$ & $\begin{array}{r}483000 \\
436000 \\
47000\end{array}$ & $\begin{array}{c}910000 \\
- \\
-\end{array}$ & $\begin{array}{c}220000 \\
- \\
-\end{array}$ & $\begin{array}{c}690000 \\
- \\
-\end{array}$ \\
\hline
\end{tabular}


Répartition des entreprises selon la récolte en 1983

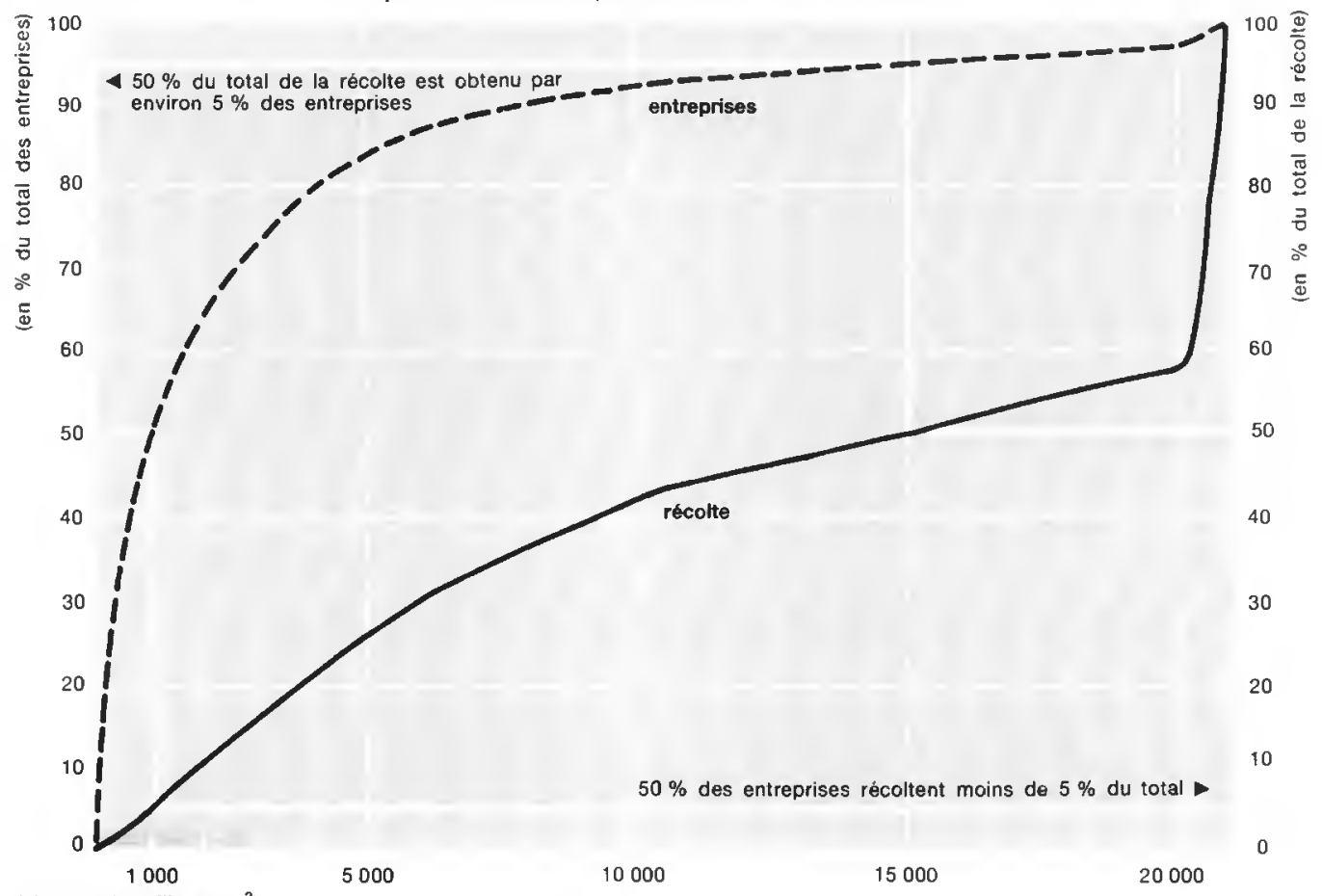

(Classes de taille en $\mathrm{m}^{3}$ )

ANNEXE IX

Activité de scierie

Répartition des entreprises selon la production en 1983

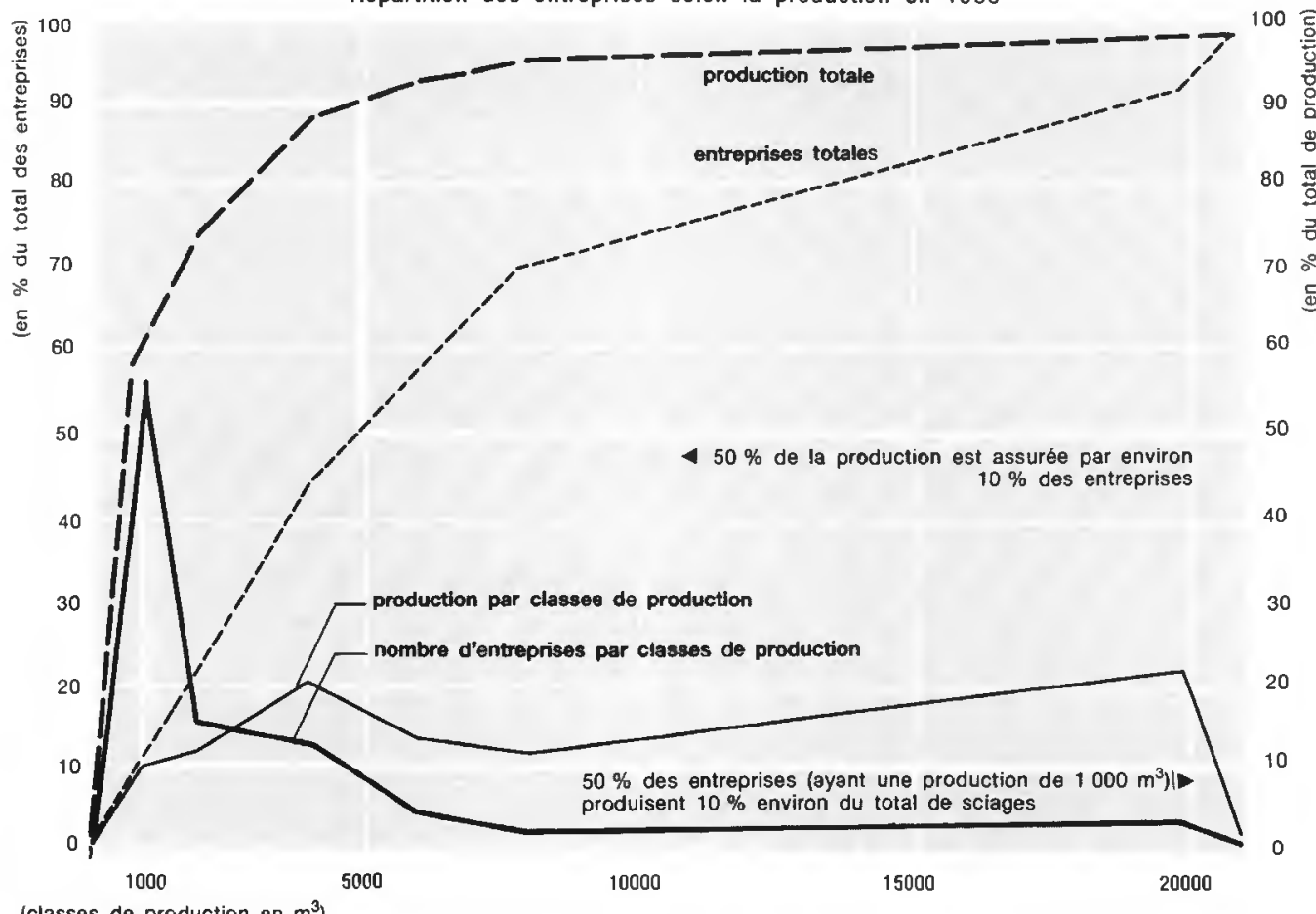

(classes de production en $\mathrm{m}^{3}$ ) 
Ouantités livrées ventilées par groupes de produits

\begin{tabular}{|c|c|c|c|}
\hline & 1982 & 1983 & 1984 \\
\hline Contreplaqués $\left(\mathrm{m}^{3}\right) \ldots \ldots \ldots \ldots \ldots \ldots$ & 443519 & 484243 & 477636 \\
\hline Panneaux de particules $\left(\mathrm{m}^{3}\right)$ & 1649558 & 1581771 & 1532828 \\
\hline Panneaux de fibres $(t)$ & 184499 & 231946 & 223752 \\
\hline Portes et fenêtres (vantaux) $\ldots \ldots \ldots \ldots \ldots \ldots$ & 7779463 & 7187265 & 6918493 \\
\hline Portes planes (unitès) $\ldots \ldots \ldots \ldots \ldots \ldots \ldots$ & 8396865 & 8150576 & 6221555 \\
\hline Charpentes $\left(\mathrm{m}^{3}\right) \quad \ldots \ldots \ldots \ldots \ldots \ldots \ldots \ldots$ & 200645 & 225202 & 206562 \\
\hline Parquets, lambris et panneaux pour parquets $\left(m^{2}\right) \ldots$ & 23750487 & 27293175 & 28893797 \\
\hline Emballages (t) . . & 903500 & 891600 & 952500 \\
\hline
\end{tabular}

Généralités sur le secteur Travail mécanique du bois

\begin{tabular}{|c|c|c|c|}
\hline $\begin{array}{c}\text { Nombre de salariés } \\
\text { par entreprise }\end{array}$ & $\begin{array}{c}\text { Nombre total } \\
\text { d'entreprises } \\
\text { de + de } 20 \text { salariés }\end{array}$ & Effectif & $\begin{array}{l}\text { Chiffre d'affaires } \\
\text { (en millions de F) }\end{array}$ \\
\hline $20-49 \ldots \ldots \ldots \ldots \ldots$ & 479 & 16187 & 5239,2 \\
\hline $50-99 \ldots \ldots \ldots \ldots \ldots$ & 163 & 11708 & 4058,6 \\
\hline $100-199 \ldots \ldots \ldots \ldots$ & 84 & 11800 & 4444,2 \\
\hline $200-499 \ldots \ldots \ldots \ldots$ & 29 & 8211 & 3942,6 \\
\hline 500 et plus $\ldots \ldots \ldots \ldots$ & 3 & 8733 & 3581,4 \\
\hline TOTAL $\ldots \ldots \ldots \ldots \ldots \ldots$ & 758 & 56639 & 21266,0 \\
\hline
\end{tabular}

Source : Enquête Annuelle d'Entreprise, 1983

ANNEXE XI

\begin{tabular}{|c|c|c|c|}
\hline $\begin{array}{c}\text { Nombre de salariés } \\
\text { par entreprise }\end{array}$ & $\begin{array}{c}\text { Nombre total } \\
\text { d'entreprises } \\
\text { de }+ \text { de } 20 \text { salariés }\end{array}$ & Effectif & $\begin{array}{l}\text { Chiffre d'affaires } \\
\text { (en millions de F) }\end{array}$ \\
\hline $20-49 \ldots \ldots \ldots \ldots$ & 585 & 18310 & 5075,7 \\
\hline $50-99 \quad \ldots \ldots \ldots \ldots \ldots$ & 182 & 12769 & 3808,5 \\
\hline $100-199 \ldots \ldots \ldots \ldots$ & 123 & 16673 & 5426,8 \\
\hline $200-499 \ldots \ldots \ldots \ldots$ & 54 & 14982 & 5146,9 \\
\hline 500 et plus $\ldots \ldots \ldots \ldots$ & 15 & 12686 & 4050,6 \\
\hline $\begin{array}{l}\text { Donneurs d'ordres (tailles } \\
\text { non significatives) } \quad \ldots \ldots \ldots \text {. }\end{array}$ & 3 & 234 & 36,3 \\
\hline TOTAL $\ldots \ldots \ldots \ldots \ldots$ & 962 & 75654 & 23544,8 \\
\hline
\end{tabular}

Source: Enquête Annuelle d'Entreprise, 1983. 


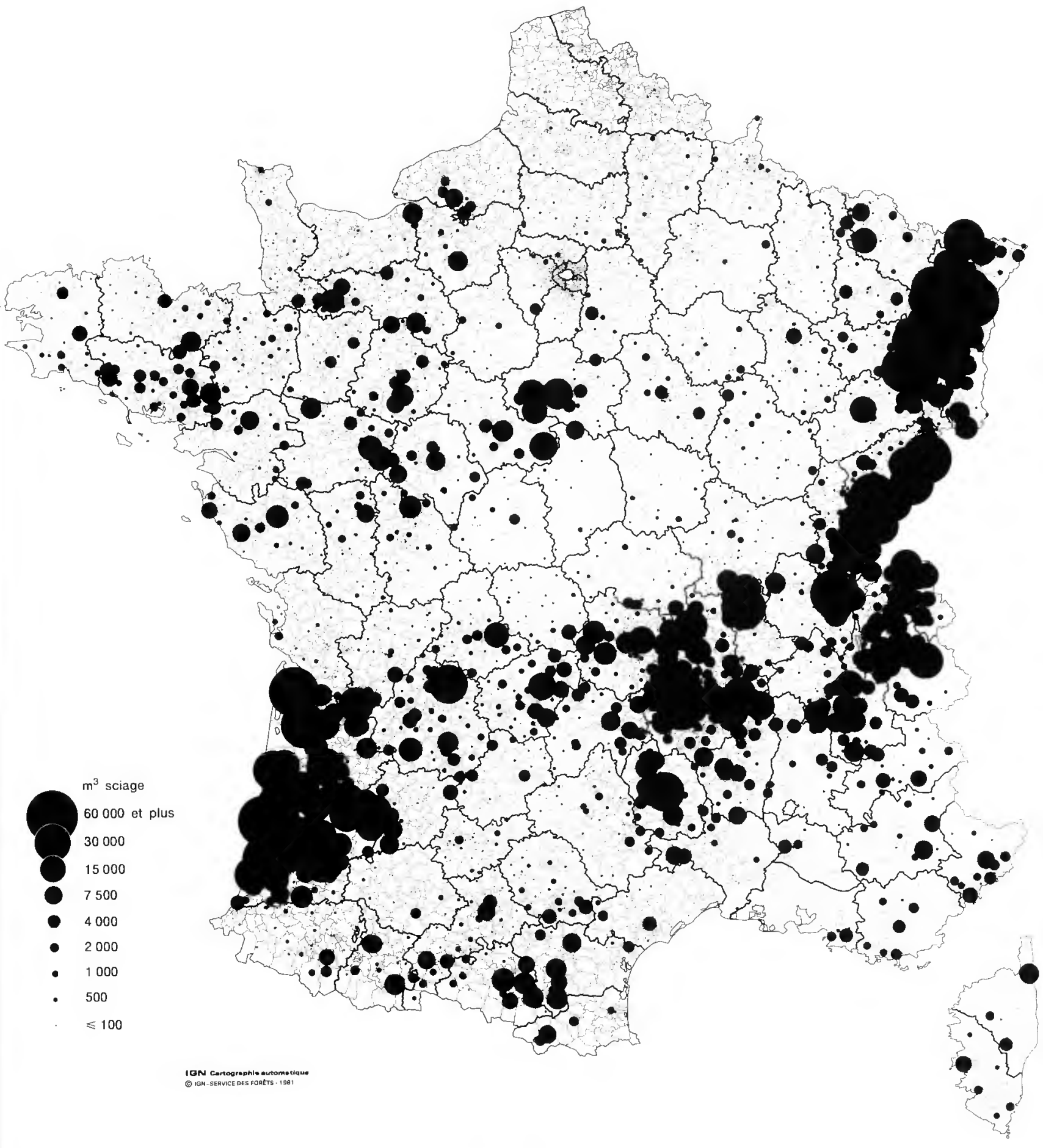




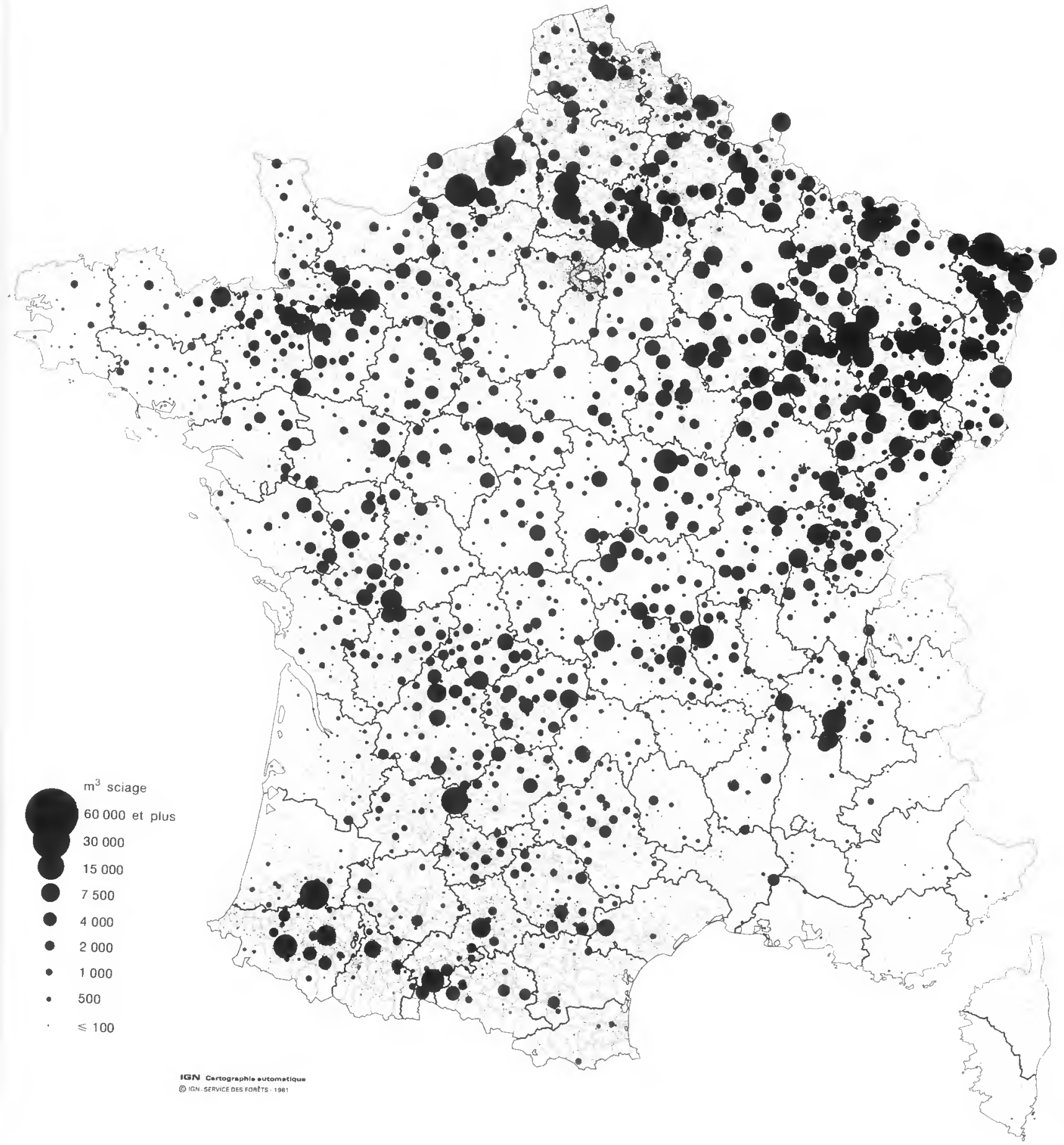


Secteur pâtes toutes entreprises

(Centre d'études et de productivité des industries des papiers, cartons et celluloses, 1983)

Nombre d'entreprises: 21

Effectif : 4950

Production totale: 1870 milliers de tonnes

Consommation de bois : 10451000 stères

Valeur HT des pâtes produites: 4631 millions de $F$.

Secteur papiers-cartons

\begin{tabular}{|c|c|c|c|}
\hline $\begin{array}{c}\text { Nombre de salariés } \\
\text { par entreprise }\end{array}$ & $\begin{array}{c}\text { Nombre total } \\
\text { d'entreprise } \\
\text { de de 20 salariés }\end{array}$ & Effectif & $\begin{array}{c}\text { Chiffre d'Affaires } \\
\text { (millions de F) }\end{array}$ \\
\hline $20-49$ & 260 & 8763 & 3774 \\
\hline $50-99$ & 144 & 10824 & 4720 \\
\hline $100-199$ & 107 & 15000 & 7560 \\
\hline $200-499$ & 90 & 27685 & 15175 \\
\hline 500 et plus & 36 & 38188 & 24677 \\
\hline TOTAL & 635 & 100460 & 55906 \\
\hline
\end{tabular}

Source: Enquête Annuelle d'Entreprise, 1983.

La statistique engloble sous ce titre les secteurs suivants: pâtes, papiers-cartons, articles de papeterie, transformation du papier, cartons ondulés, cartonnages. 


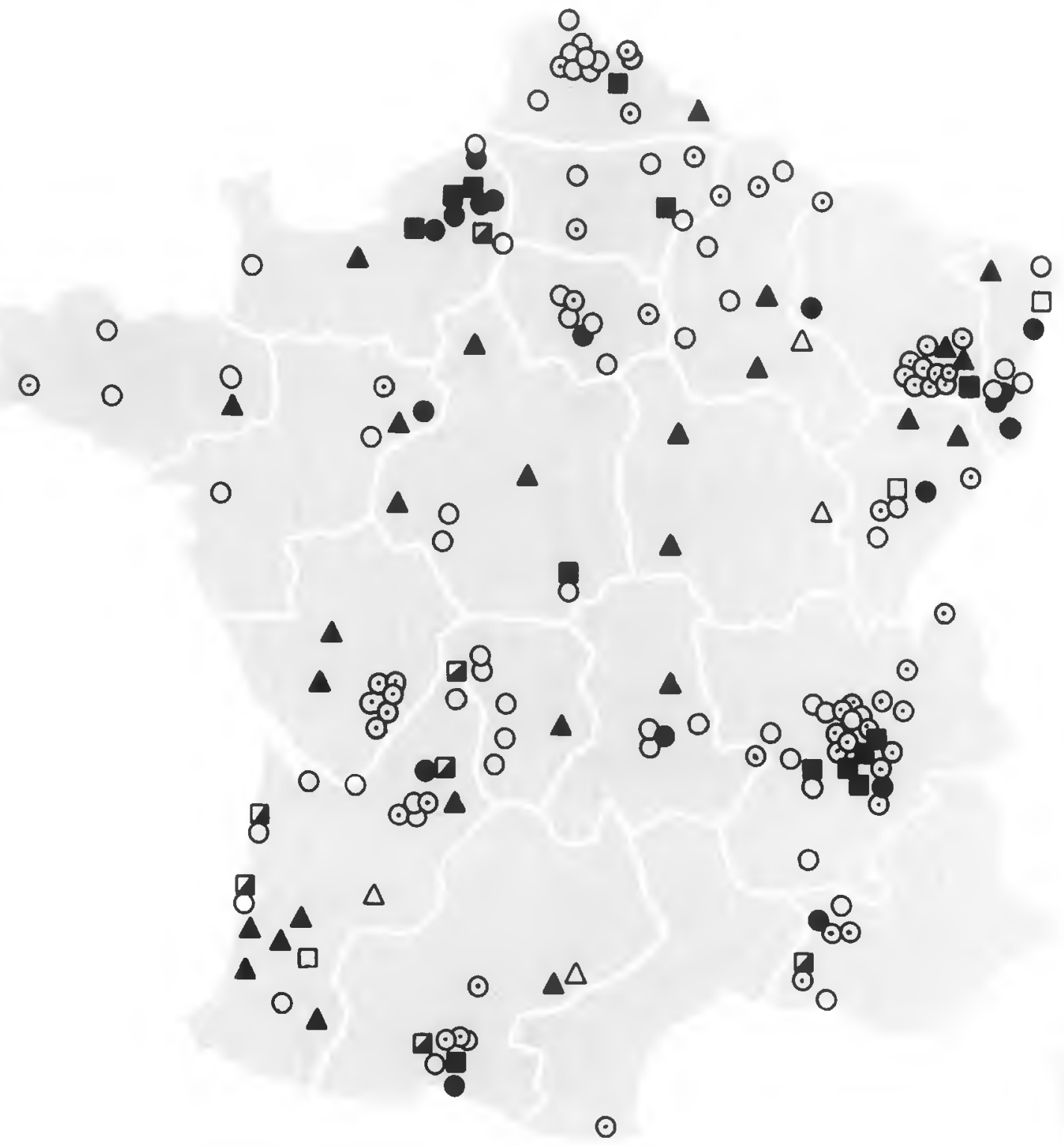

A panneaux de particules

$\triangle$ panneaux de fibres

pâtes mècaniques ou mi-chimiques

pâtes au sulfate

pâtes au sulfite cartons-emballages

- papier journal ou à usage graphique

$\odot$ papiers-cartons

d'après Centre d'Etudes et de Praductivité des industries des papiers, cartons 


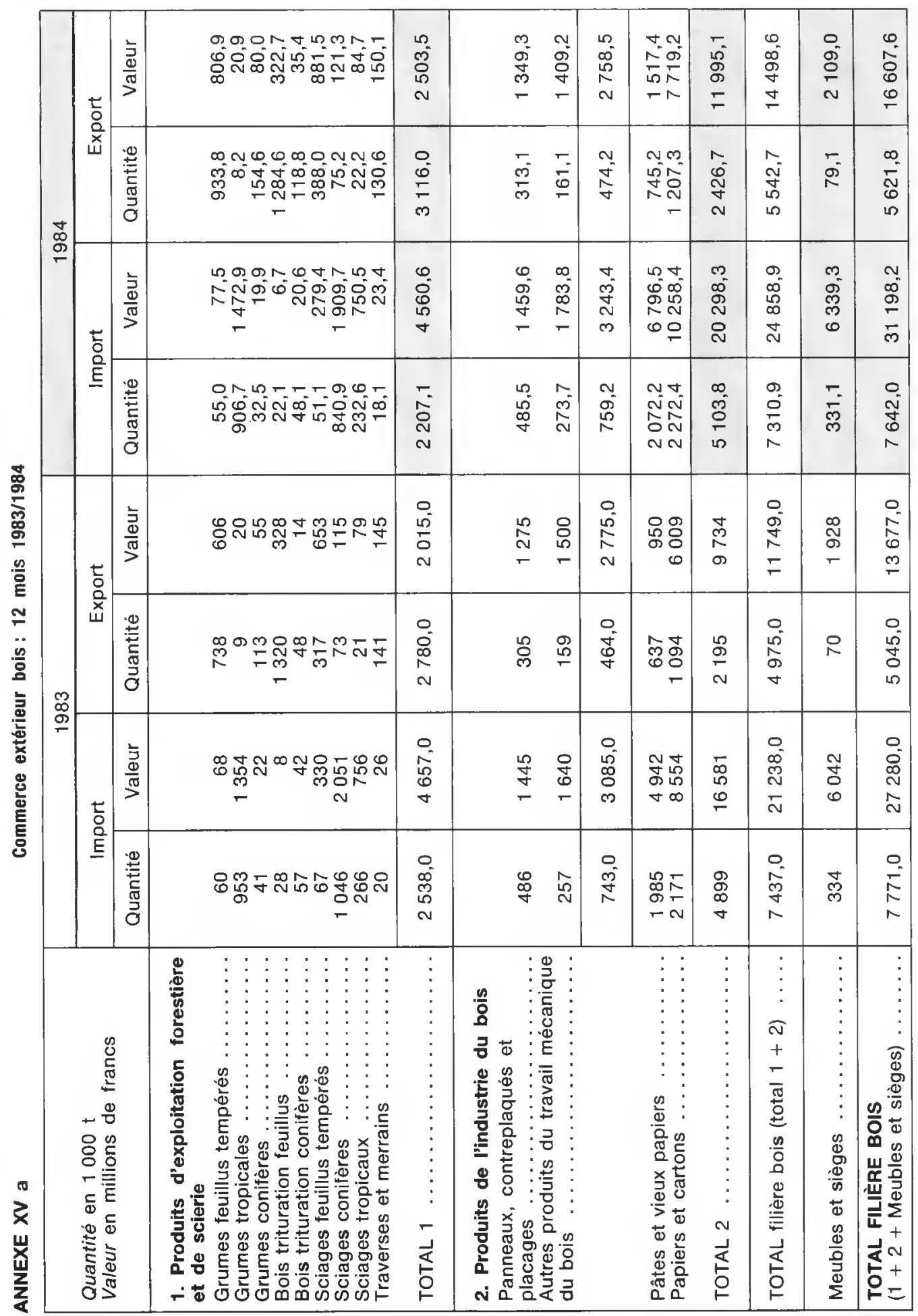




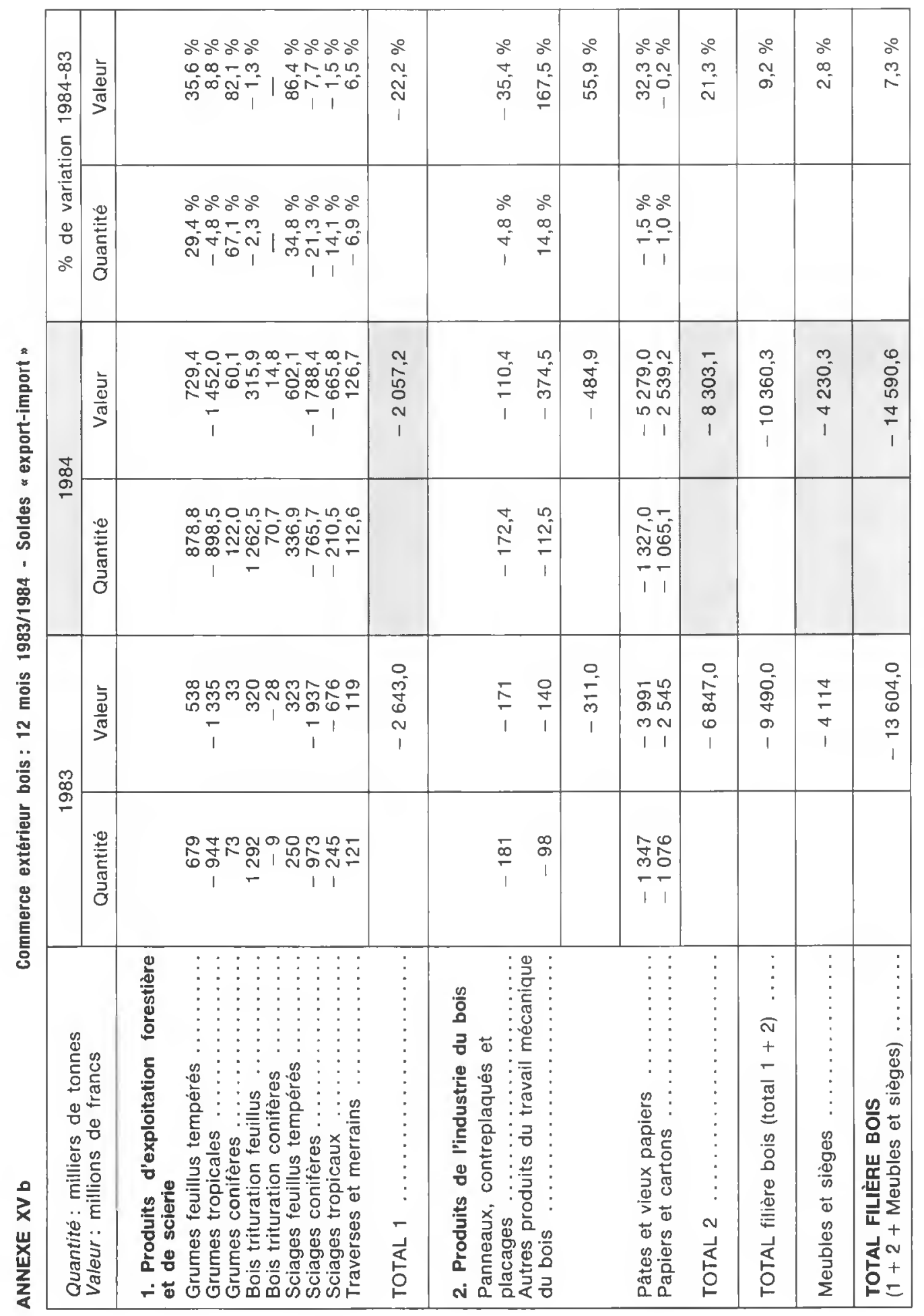




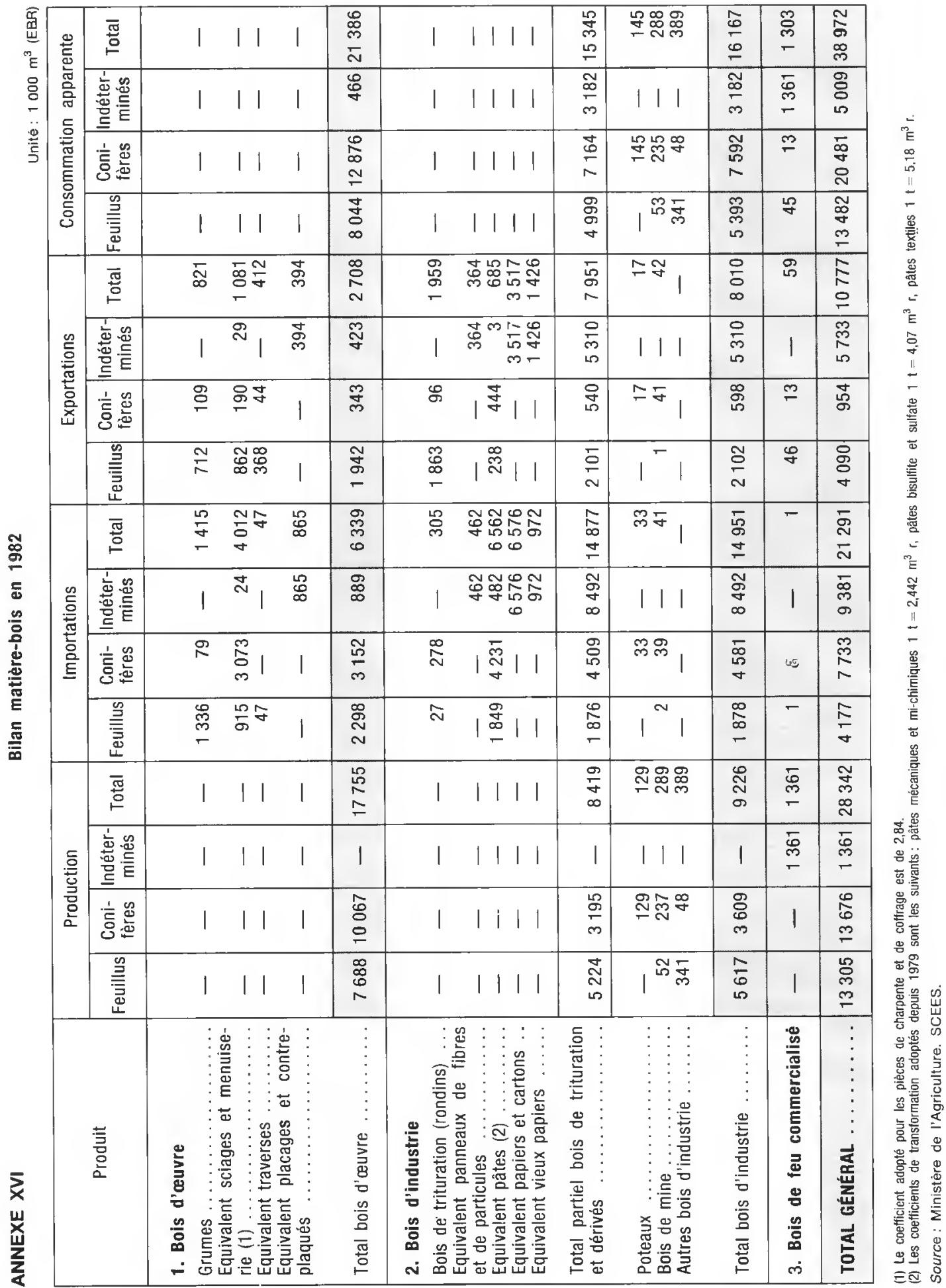




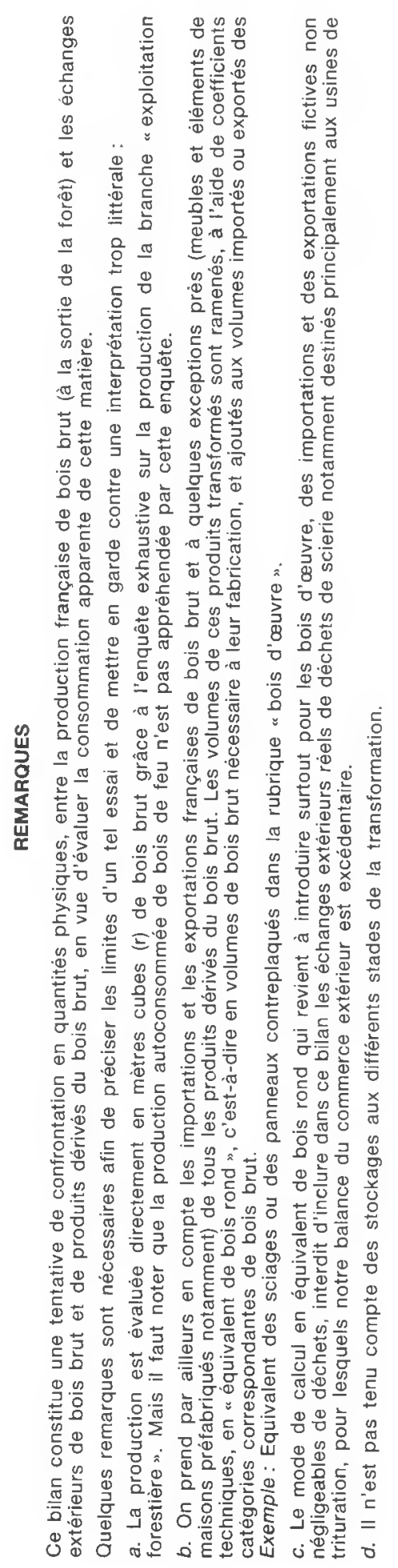




\section{ADMINISTRATIONS ET ÉTABLISSEMENTS PUBLICS}

Ministère de l'Agriculture

Secrétariat d'Etat à l'Agriculture et à la Forêt

Direction des Forêts

Ministère du Commerce, de l'Artisanat et du Tourisme

Ministère de l'Environnement, Direction de la Protection de la Nature

Ministère de la Recherche et de la Technologie

Ministère du Redéploiement industriel et du Commerce extérieur

- Sous-Direction bois, papier, ameublement

- Direction générale de l'énergie et des matières premières

Ministère de I'Urbanisme, du Logement et des Transports,

Direction de la construction

Agence française pour la maîtrise de l'énergie

Office national des forêts (O.N.F.)

Ecole française de Papeterie

Ecole nationale du Génie rural des Eaux et des Forêts

Ecole nationale des Ingénieurs des Travaux des Eaux et des Forêts

Ecole supérieure du Bois

Institut des Sciences et Techniques du Bois (I.S.T.B.)
78, rue de Varenne, 75007 Paris.

(1) 555.95 .50

78 , rue de Varenne, 75007 Paris

(1) 555.95 .50

1 ter, avenue de Lowendal, 75007 Paris

80 , rue de Lille, 75007 Paris

(1) 555.95 .50

(1) 556.24 .24

14, boulevard du Général-Leclerc, 92524 Neuilly Cedex

1, rue Descartes, 75231 Paris Cedex 05

(1) 758.12 .12

(1) 634.33 .33

101, rue de Grenelle, 75007 Paris

(1) 556.36 .36

32, rue de Guersant, 75840 Paris Cedex 17

(1) 572.85 .85

101, rue de Grenelle, 75700 Paris

(1) 556.36 .36

2 bis, avenue du Parc-de-Passy

75775 Paris Cedex 16

27, rue Louis-Vicat, 75015 Paris

(1) 503.91 .92

(1) 765.20 .00

2, avenue de Saint-Mandé

75570 Paris Cedex 12

Voie $8, n^{\circ} 55$. Domaine universitaire, B.P. 65,38402 Saint-Martin d'Hères

Centre de Paris

19. avenue du Maine, 75732 Paris Cedex 15

Centre de Nancy

14, rue Girardet, 54042 Nancy Cedex

Centre de Montpellier

Domaine de Lavalette, avenue du Val-deMontferrand, 's4060 Montpellier Cedex

Domaine des Barres, 45290 Nogent-sur-Vernisson

6, avenue de Saint-Mandé, 75012 Paris

Route du Fort-de-la-Voivre, 88000 Epinal

(1) 346.11 .68

(76) 42.01 .27

(1) 544.38 .86

(8) $335,10.20$

(67) 54.46 .96

(38) 97.60 .20

(1) 628.09 .33

(29) 34.02 .87

(1) 562.63 .16

(6) 408.41 .98

(1) 280.69 .77

85 , rue Lafayette, 75009 Paris

(1) 260.64 .12

1, place Andrè-Malraux, 75001 Paris

(38) 97.60 .59

Domaine des Barres, 45290 Nogent-sur-Vernisson

10, avenue de Saint-Mandé, 75012 Paris

(1) 344.06 .20

45 bis, avenue de la Belle-Gabrielle,

(1) 873.32 .95

94130 Nogent-sur-Marne

B.P. 7110,38020 Grenoble Cedex

(76) 44.82 .36

23, avenue Bosquet, 75007 Paris

(1) 555.23 .49

Département des Recherches forestières Centre de Recherches d'Orléans, Ardon, 45160 Olivet
(38) 63.02 .06 
Institut national de la recherche agronomique (I.N.R.A.) (suite)

Laboratoire d'économie forestière et agricole

\section{ORGANISATIONS SYNDICALES}

Association France Promobois Construction Association nationale des Centres régionaux de la proprièté forestière (A.N.C.R.P.F.)

Compagnie nationale des ingénieurs et ex perts forestiers

Confèderation nationale des industries $\mathrm{du}$ bois (C.N.I.B.)

Confédération française de l'industrie des papiers, cartons et cellulose

(C.O.P.A.C.E.L.)

Conseil interfédèral du bois (C.I.B.)

Fédération française du commerce du bois

Féderation française des importateurs de bois du Nord

Féderation française des bois tropicaux et americains

Fédération française du négoce de bois d'œuvre et de produits dèrivés

Chambre syndicale nationale des agents en bois et dérives

Fèderation des industries lourdes du bois (F.I.L.B.)

Fédération nationale du bois (F.N.B.)

Fèderation nationale des communes forestières de France (C.O.F.O.R.)

Fèderation nationale des industries du bois pour le bâtiment

Fèdération nationale de l'injection des bois (F.N.I.B.)

Fédèration nationale des Syndicats de propriétaires forestiers sylviculteurs

Fédération des Syndicats de producteurs de pâtes pour papiers et textiles

Syndicat des fabricants de papier-journal

Syndicat gènéral des acheteurs-utilisateurs de bois de papeterie

Syndicat général des acheteurs-utilisateurs de bois pour panneaux

Syndicat national des constructeurs de charpentes en bois lamellè-collé

Union nationale française des Chambres syndicales de charpente, menuiserie et parquets

Union nationale des industries françaises de l'ameublement (U.N.I.F.A.)

Union nationale des industries françaises de l'emballage utilisant le bois (U.N.E.B.)

Union des syndicats de constructeurs de maisons individuetles
Stations de Recherches forestières d'Avignon (90) 89.33 .25 Avenue A.-Vivaldi, 84000 Avignon

Stations de Recherches forestieres de Bordeaux

(56) 07.63 .06

Domaine de l'Hermitage, Pierroton. 33610 Cestas Principal

Centre de Recherches forestieres Champenoux, 54280 Seichamps

332.09 .21

14, rue Girardet, 54042 Nancy Cedex

(8) 335.43 .06

10, rue Riquet, 75019 Paris

(1) 245.90 .08

34, rue Hamelin, 75116 Paris

(1) 720.68 .15

6, avenue de Saint-Mandé, 75012 Paris

(1) 341.02 .09

36, avenue Hoche, 75008 Paris

(1) 561.00 .90

154, boulevard Haussmann, 75008 Paris

(1) 562.70 .55

36, avenue Hoche, 75008 Paris

(1) 561.00 .90

8, rue du Colonel-Moll, 75017 Paris

(1) 267.64 .75

30, avenue Marceau, 75008 Paris

(1) 720.17 .32

1, place André-Malraux, 75001 Paris

(1) 260.30 .27

13, rue du Général-Bertrand, 75007 Paris

(1) 567.47 .98

36, avenue Hoche, 75008 Paris

(1) 561.00 .90

36, avenue Hoche, 75008 Paris

(1) 561.00 .90

6. rue de la Trèmoille, 75008 Paris

(1) 720.36 .32

164, boulevard Haussmann, 75008 Paris

(1) 562.63 .16

156, boulevard Haussmann, 75008 Paris

(1) 562.82 .55

164, boulevard Haussmann, 75008 Paris

(1) 562.63 .16

30, avenue Marceau, 75008 Paris

(1) 720.17 .32

9. rue La Pérouse, 75784 Paris Cedex 16

(1) 720.10 .20

10, rue du Dèbarcadère

(1) 574.99 .42

75852 Paris Cedex 17

(1) 628.68 .61

57, rue de Clichy, 75009 Paris

(1) 282.16 .00

6, rue Paul-Valéry, 75116 Paris

(1) 720.10 .20 